OPEN ACCESS

Edited by:

Vidhya Chakrapani,

Rensselaer Polytechnic Institute,

United States

Reviewed by:

Jose Solla-Gullon,

University of Alicante, Spain

Wei Wang

Nanjing University, China

${ }^{*}$ Correspondence:

Kristina Tschulik

Kristina.tschulik@rub.de

Specialty section:

This article was submitted to Catalysis and Photocatalysis, a section of the journal

Frontiers in Chemistry

Received: 09 September 2019 Accepted: 16 December 2019 Published: 17 January 2020

Citation:

Wonner K, Rurainsky C and Tschulik K (2020) Operando Studies of the Electrochemical Dissolution of Silver Nanoparticles in Nitrate Solutions Observed With Hyperspectral Dark-Field Microscopy. Front. Chem. 7:912 doi: 10.3389/fchem.2019.00912

\section{Operando Studies of the Electrochemical Dissolution of Silver Nanoparticles in Nitrate Solutions Observed With Hyperspectral Dark-Field Microscopy}

\author{
Kevin Wonner, Christian Rurainsky and Kristina Tschulik* \\ Chair of Analytical Chemistry II, Faculty of Chemistry and Biochemistry, Ruhr University Bochum, Bochum, Germany
}

Since nanoparticles are frequently used in commercial applications, there is a huge demand to obtain deeper insights into processes at the nanoscale. Especially, catalysis, chemical and electrochemical reaction dynamics are still poorly understood. Thus, simultaneous and coupled opto-and spectro-electrochemical dark-field microscopy is used to study in situ and operando the electrochemically driven dissolution mechanism of single silver nanoparticles in the presence of nitrate ions as non-complexing counter-ions, herein. Hyperspectral imaging is used to probe the intrinsic localized surface plasmon resonance of individual silver nanospheres before, during and after their electrochemical oxidation on a transparent indium tin oxide (ITO) electrode. Furthermore, optical video imaging was performed for additional information. Based on the complete loss of spectral information and intensity, a dissolution of the particles during the reaction was concluded. This way it is revealed that the dissolution of individual particles proceeds over several seconds, indicating a hindrance by the nitrate ions. Only electrochemical analysis does not provide this insight as the measured current does not allow distinguishing between successive fast dissolution of one particle after another or slow dissolution of several particles in a concerted manner. For comparison, experiments were performed in the presence of chloride ions. It was observed that the silver chloride formation is an instantaneous process. Thus, it is possible to study and define the reaction dynamics on the single nanoparticle level in various electrochemical systems and electrolyte solutions. Accordingly, operando opto- and spectro-electrochemical studies allow us to conclude, that the oxidation of silver to solvated silver cations is a kinetically slow process, while the oxidation to silver chloride is fast. We propose this approach as a new method to study electrocatalyst materials, their transformation and degradation under operando conditions.

Keywords: dark-field microscopy, silver nanoparticle, hyperspectral dark-field microscopy, operando spectroscopy, plasmon resonance, single entity electrochemistry 


\section{INTRODUCTION}

Nanomaterials, like small nanoparticles (NP), show a high surface area to volume ratio and an altered electronic structure, as compared to their bulk counterparts (Kreibig, 1974; Zhu et al., 2013). Due to the tunable and often advantageous characteristics of those nano-entities they are widely used for industrial application (Cheng and Compton, 2014; Sokolov et al., 2015). For instance, silver nanoparticles (AgNPs) are applied in products like mobile phones, refrigerators, energy storage and batteries or in cosmetics, medicine and sportswear due to their antibacterial and anti-inflammatory properties (Cheng et al., 2013; Chernousova and Epple, 2013; Tschulik et al., 2013; Yang et al., 2013).

Catalytic processes in heterogeneous catalysis occur at the surface of the catalyst material. Especially, noble metal nanoparticles are often used as electrocatalyst and due to their high surface energies only a small amount of material is needed (Welch and Compton, 2006; Salehi-Khojin et al., 2013; Cao et al., 2015; Wang et al., 2015; Strickler et al., 2017; Konopatsky et al., 2018; El Arrassi et al., 2019; Evers et al., 2019). Electrochemical reductions and oxidations can be triggered in a deliberate way by application of a defined potential at the electrode and thus, the surface of the catalyst material (Shan et al., 2012). Even though nanoparticles, and in particular AgNPs, were intensively investigated with electrochemical and chemical methods, there is still a lack of knowledge about their behavior in solution, reactivities, and reaction mechanisms (Wong et al., 2009; Ngamchuea et al., 2018). Often, catalytic reaction pathways are also only poorly understood. Therefore, there is a huge demand to get deeper insights into reaction mechanisms, especially for electrochemically (and thus potential) driven reactions, at and of nanostructures on the single entity nanomaterial level. The latter one is of special interest, because even small changes in the size and shape of the materials (which likely alters by the synthesis) strongly influence and vary their electrochemical and physical behavior (Roduner, 2006; Viñes et al., 2014; Khan et al., 2017). For those purposes additional operando analytical, optical and spectroscopic methods are needed to get supplementary and time-dependent information to the electrochemical current signals.

Today, a huge variety of analytical methods is established, which are capable of studying the structure of nanomaterials ex situ under harsh conditions, like vacuum. Examples for such devices are scanning electron microscopy (SEM), transmission electron microscopy, scanning tunneling microscopy and atomic force microscopy (Binnig and Rohrer, 1983; Wang, 2000; Ferreira et al., 2015). Those methods offer a precise analysis (up to atomic resolutions) of shapes and sizes of nanostructures. However, they do not allow studying their real behavior in solution. In contrast to that it is also possible to use in situ methods, like dynamic light scattering or UV/VIS spectroscopy (Haiss et al., 2007; Amendola and Meneghetti, 2009; Ramos, 2017). Although it is feasible to measure nano-entities within their dispersion media, obtainable results are inaccurate and provide only imprecise size distributions. Moreover, it is only possible to guess the shapes by the evaluation of measured data. Instead of this, electrochemical methods can be used to study even single particles quantitatively during an electrochemical reaction in situ in various aqueous electrolytes (nanoparticle impact) (Tschulik et al., 2014). If a NP is in contact with a polarized electrode, it can undergo several chemical reactions, like complete conversion of the particle itself (Batchelor-McAuley et al., 2015; Saw et al., 2018) or catalytically enhanced reactions at the particle surfaces (Cheng and Compton, 2014).

Although particles sizes (Batchelor-McAuley et al., 2015), compositions (Saw et al., 2016), reaction kinetics (Saw et al., 2017), reaction layer distances (Little et al., 2018), or diffusion coefficients (Saw et al., 2018) can be determined by NP impacts their reactions mechanism and additionally processes at the surfaces are today poorly understood and still under debate. Despite working with similar experimental conditions, different results were obtained. Thus, various mechanisms of electrochemical single nanoparticle conversion were suggested (Cheng and Compton, 2014; Oja et al., 2017; Robinson et al., 2017; Saw et al., 2017; Ustarroz et al., 2017; Kanokkanchana et al., 2018). Since electrochemistry is limited by the amount of charge or current as the information that can be obtained from the measurements, additional non-electrochemical methods are needed to gain supplementary information. For instance, spectroscopy can be implemented and coupled with electrochemical methods to get in situ and operando information of a nanoparticle during its reaction.

A huge variety of those opto- and spectro coupled electrochemical methods has been reported in literature, like surface-enhanced Raman spectroscopy (Sherry et al., 2005; Stiles et al., 2008; Weber et al., 2015), electrogenerated chemiluminescence-imaging (Wilson et al., 2015) or singleparticle fluorescence microscopy (Hao et al., 2017; Tanner et al., 2017). Metal nanoparticles, like silver and gold, exhibit an intrinsic localized surface plasmon resonance (LSPR), which is defined by the confinement of the size and the resulting electronic structure (Wilson and Willets, 2016). A representative illumination of the LSPR of a spherical nanoparticle is shown in Figure 1. The confined electrons in the conduction band, the electron cloud, are displaced by an incoming electric field (light) (Wilson and Willets, 2016). The negatively charged electron cloud is withdrawn again, due to the Coulomb forces of the remaining fixed and positively charged nuclei (Willets et al., 2017). Based on this coherent oscillation, incoming light with a larger wavelength compared to the size of the noble metal nanoparticles interact with those particles. Thus, light is scattered dependent on the incoming wavelength in relation to the size of the particle. Moreover, the LSPR depends on the shape, dielectric environment (surrounding media) and particle composition (Mock et al., 2002; Kelly et al., 2003; Wilson and Willets, 2016; Willets et al., 2017).

The excitation energy and intensity of the localized surface plasmon resonance of nanomaterials can be probed with plasmon-based electrochemical current microcopy (PECM) and particularly with dark-field microscopy (DFM). The latter is able to precisely study the spectral positions and intensities of the extinction spectra (LSPR) of individual nanoparticles by hyperspectral imaging (HSI) (Willets and van Duyne, 2007; 


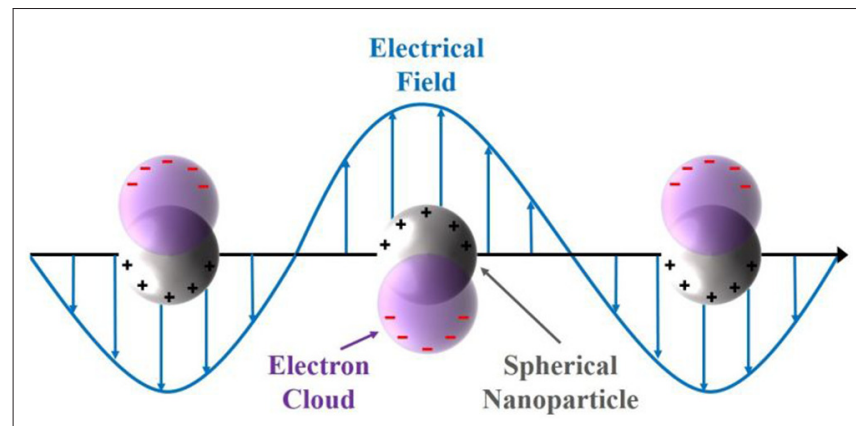

FIGURE 1 | Representation of the localized surface plasmon resonance of a spherical nanoparticle and the electron cloud displacement due to the interaction with an incoming electric field.

Batchelor-McAuley et al., 2014; Fang et al., 2014; Brasiliense et al., 2016; Sundaresan et al., 2018; Sun et al., 2018; Wonner et al., 2018, 2019; Qiu et al., 2019). In previous works of the groups of Tao (Fang et al., 2014), Kanoufi (Batchelor-McAuley et al., 2014; Brasiliense et al., 2016), and our group (Wonner et al., 2019) silver nanoparticles were dissolved electrochemically in the presence of thiocyanate anions with PECM and DFM, respectively. A drawback of the PECM is that only intensities can be tracked during the reaction, whereas hyperspectral DFM can observe the spectral changes during a particle reaction. Moreover, previous works showed that it is even possible to resolve the spectral changes in the scattering spectrum of a single particle during an electrochemical oxidation by a composition change of the particle itself (Wonner et al., 2018).

Unexpectedly, although nearly the same electrochemical conditions are used for the oxidation of silver nanoparticles in the presence of nitrate, there is still controversy about the oxidation mechanism (Cheng and Compton, 2014; Patel et al., 2015; Oja et al., 2017; Saw et al., 2017; Ustarroz et al., 2017). On the one hand, the oxidation is described as an instantaneous and full dissolution, while on the other hand an incomplete oxidation with a continuous dissolution mechanism has been reported. For this purpose, hyperspectral dark-field microscopy (HSI-DFM) should be employed to study the dissolution process for detailed insight into the reaction dynamic of small nano-entities.

In this work, the oxidation process of silver nanoparticles is studied to figure out the mechanism of the oxidation in the presence of nitrate anions $\left(\mathrm{NO}_{3}^{-}\right)$of the electrolyte solution, which is illustrated in Figure 2. Moreover, the experiments should additionally be carried out in the presence of chloride $\left(\mathrm{Cl}^{-}\right)$anions to compare the reaction dynamics in the presence of two different anions (Figure 2), a complexing and a non-complexing anion. For reactions in chloride solution, an instant oxidation, and thus color change is expected with a defined spectral shift. For the two electrolytes, the reaction is given in Equation (1) and (2), respectively.

$$
\begin{aligned}
\mathrm{Ag}(s)+\mathrm{NO}_{3}^{-}(a q) & \rightleftharpoons \mathrm{Ag}^{+}(a q)+e^{-}+\mathrm{NO}_{3}^{-}(a q) \\
\mathrm{Ag}(s)+\mathrm{Cl}^{-}(a q) & \rightleftharpoons \mathrm{AgCl}(s) \downarrow+e^{-}
\end{aligned}
$$

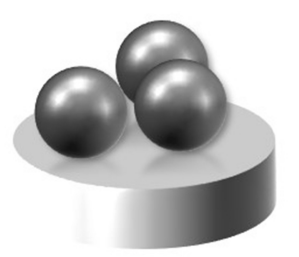

AgNPs at electrode
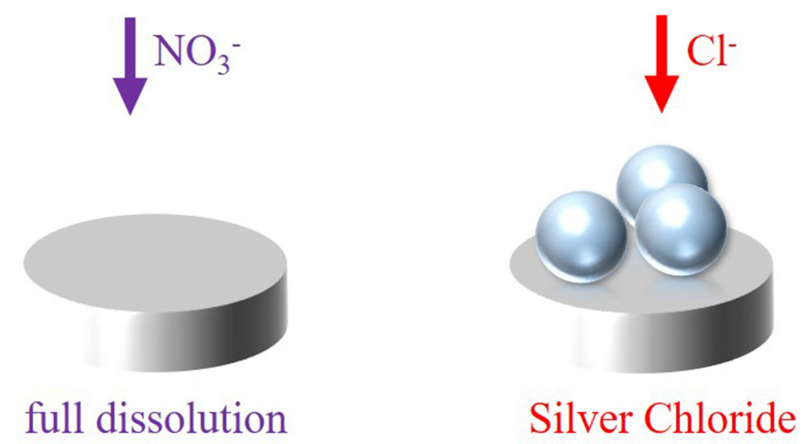

FIGURE 2 | Schematic representation of the electrochemical oxidation of silver nanoparticles in the presence of either nitrate or chloride anions at a polarized electrode.

In the presence of non-complexing nitrate ions, silver will be oxidized to silver cations $\left(\mathrm{Ag}^{+}\right)$, which are dissolved in the electrolyte solution (Equation 1). Hence, a decrease in intensity and loss of spectral information is expected during the oxidation of the particles. From the dissolution rate, conclusions about the mechanism of the electrochemical oxidation can be drawn. In contrast, the formation of silver chloride $(\mathrm{AgCl})$ is thermodynamically driven in the presence of chloride ions, which favors the oxidation of the silver nanoparticles and shifts it to lower potentials (Equation 2) (Toh et al., 2013; Saw et al., 2018). The reaction is supposed to proceed in a fast way, which can be observed by an instantaneous spectral change. Moreover, an intensity alteration of the scattered light is supposed to happen, too (Brasiliense et al., 2018).

\section{EXPERIMENTAL PART}

\section{Synthesis of Silver Nanoparticles}

Fifty milliliters of ultra-pure water were heated in a $100 \mathrm{~mL}$ round bottom flask until boiling. Five hundred microliters of $10 \mathrm{mM} \mathrm{AgNO} 3$ (Alfa Aesar, 99.9995\% metal basis) solution followed by $500 \mu \mathrm{L}$ of a $60 \mathrm{mM}$ tri-sodium citrate (AnalR NORMAPUR, VWR chemicals) solution were quickly added under vigorous stirring. The mixture was boiled under reflux for $1 \mathrm{~h}$ and no color change was observed. After that, first 500 $\mu \mathrm{L}$ of the $60 \mathrm{mM}$ tri-sodium-citrate solution and subsequently 2 mg D-(+)-glucose (AnalR NORMAPUR, VWR chemicals) were added to the mixture. The mixture was boiled and stirred for another $1 \mathrm{~h}$. Afterwards it was cooled down to room temperature using an ice bath and later centrifuged for $20 \mathrm{~min}$ at 20,000 
rcf and the supernatant solution was removed until about 200 $\mu \mathrm{L}$ were left, the suspension was then refilled using ultra-pure water to $2 \mathrm{~mL}$. The obtained particle suspension exhibited a concentration of roughly $0.25 \mu \mathrm{g} / \mu \mathrm{L}$. The particle size was determined with underpotential deposition of lead on silver. A size distribution of $28 \pm 3 \mathrm{~nm}$ was obtained (Grasmik et al., 2018). The used citrate served as a reducing and capping agent to prevent particle agglomeration. The electrochemical response of silvernanoparticles is not measurably affected by this small capping agent. Hence, this effect can be neglected.

\section{Chemicals and Materials}

UV-treated ultrapure water (Thermo Scientific Barnstead GenPure $x$ CAD Plus), exhibiting a conductivity of $0.055 \mu \mathrm{S} \mathrm{cm}^{-1}$ at $23^{\circ} \mathrm{C}$, was used for all experiments, aqueous solutions and cleaning processes. All used chemicals were of analytical grade. Potassium chloride $(\mathrm{KCl})$ and potassium nitrate $\left(\mathrm{KNO}_{3}\right)$ with a purity of $99.0-100.5$ and $99.999 \%$, respectively, were purchased from Sigma-Aldrich. Used indium tin oxide (ITO) coated glass slides were purchased from Sigma-Aldrich and exhibited a surface resistivity of $8-12 \Omega /$ sq. The size of the ITO-squares was $4 \mathrm{~mm}^{2}$. Gold was sputtered from a $\varnothing 57 \times 0.1 \mathrm{~mm}$ gold target disc with a purity of $99.999 \%$, which was purchased from Micro to Nano. All glassware and ITO-glass slides were sonicated for $30 \mathrm{~min}$ in a mixture of $50 \%$ acetone, $25 \%$ ethanol and $25 \%$ isopropanol. Afterwards, the procedure was repeated in pure water. All glassware was dried with an argon stream.

\section{Electrochemical Cell and Dark-Field Microscopy}

The general working principal of the dark-field microscope is shown in Figure 3A. White light is generated by a halogen lamp and then partly blocked by an aperture and focused by a condenser toward the sample (electrochemical cell). Incoming

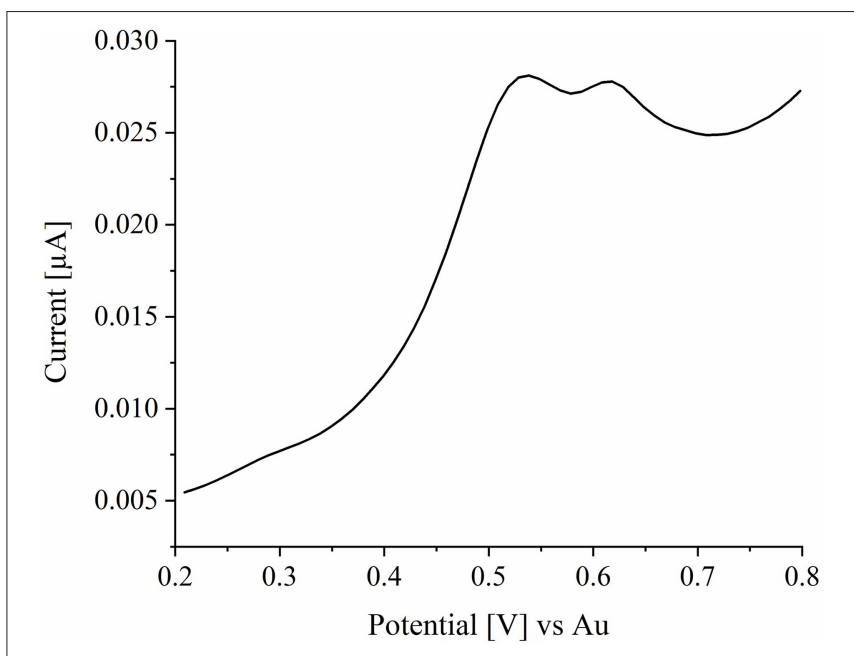

FIGURE 4 | LSV of silver nanoparticles in a $250 \mathrm{mM}$ potassium nitrate solution. The potential was swept from 0.2 to $0.8 \mathrm{~V}$ with a scan rate of $0.005 \mathrm{~V} \cdot \mathrm{s}^{-1}$.
A

\section{Dark-Field Microscope}

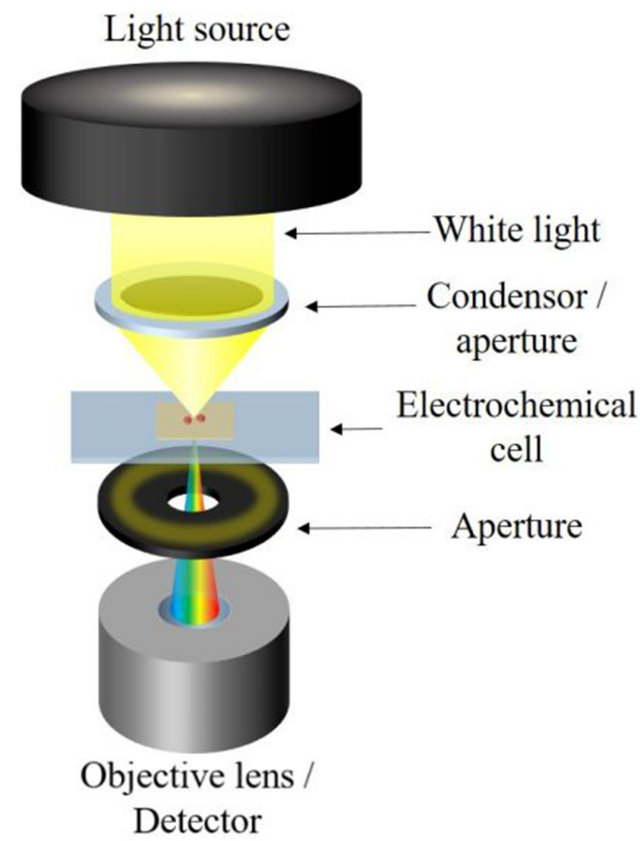

B Electrochemical Cell

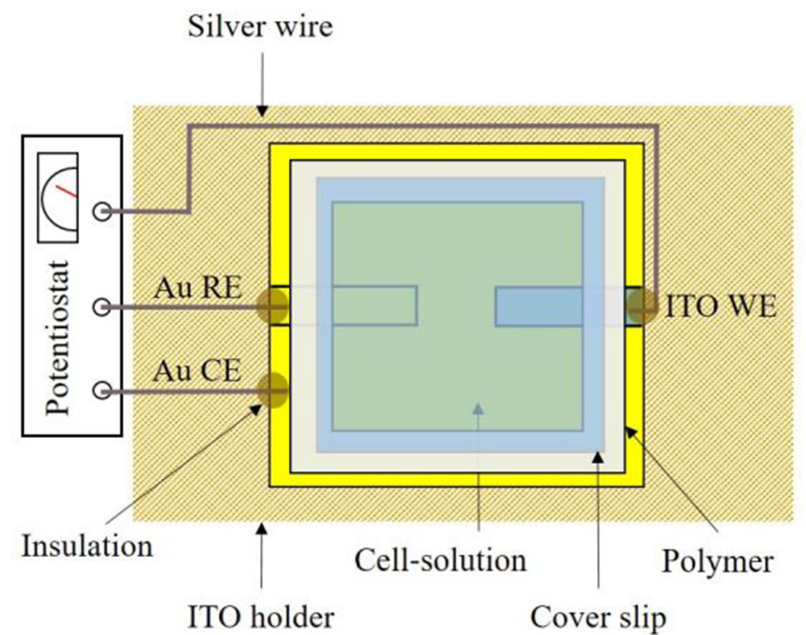

ITO holder
Cover slip

FIGURE 3 | (A) Principle setup of a dark-field microscope. (B) Designed transparent electrochemical cell for simultaneous opto- and spectro electrochemical dark-field microscopy. 
light interacts with the sample and non-scattered light is blocked by an additional aperture. Only scattered light reaches the objective lens. The light is detected by either an optical charge coupled device (CCD) camera or a hyperspectral camera. The used dark-field microscope was constructed by CytoViva Inc. and includes an Olympus BX43 Microscope. A UPL Fluorite 100x/.6-1.30 Iris Objective oil immersion objective was equipped with an illumination area of $87.5 \cdot 65 \mu \mathrm{m}$. Immersion oil ensured a contact between the condenser, sample and oil objective. For illumination, a halogen lamp and a CytoViva Optical Illuminator were used. A color Retiga R1 OEM Camera (CCD) was used for optical video imaging and a CytoViva Imaging System 2.5, consisting of a Specim ImSpector V10E Spectograph and a Cooke Pixelfly Spectral Camera, was used for hyperspectral imaging. All obtained spectra are wavelength corrected against the halogen lamp spectrum. An optical resolution down to $10-15 \mathrm{~nm}$ of plasmon active particles like silver nanospheres can be achieved.

All electrochemical experiments were performed with a PalmSens 4 (PalmSens BV) potentiostat. For the electrochemical experiments combined with dark-field microscopy, an in-house designed transparent electrochemical cell was prepared. The schematic illustration of this cell is given in Figure 3B. A threeelectrode system was used in each measurement, consisting of an ITO working electrode (WE), a gold counter electrode (CE) and a gold quasi-reference electrode (RE). All given potentials were measured against the latter one. For fabrication, a thin gold layer was sputtered on the ITO glass slide using a Sputter Coater (108auto from Cressington Scientific Instruments UK). The conductive film between each electrode was mechanically removed. The ITO-glass slide was placed into a PLA-holder and silver wires were used for electrical connection to the electrodes. Epoxy resin was used as insulation and a polymer gasket was placed on the ITO-glass slide to seal the cell. $0.3 \mu \mathrm{L}$ of the silver nanoparticles were added to the ITO WE and the particles were immobilized at the electrode and remaining liquid was removed with a tissue paper. Then $30-60 \mu \mathrm{L}$ of electrolyte solution was added and the cell was closed by a cover slip.

\section{RESULTS}

\section{Hyperspectral Dark-Field Imaging}

For the oxidation of metallic silver nanoparticles to solvated silver cations, linear sweep voltammetry was performed in a $250 \mathrm{mM}$ potassium nitrate solution. AgNPs $(0.3 \mu \mathrm{L})$ were immobilized on the ITO surface and electrolyte solution was added. The potential at the ITO WE was swept from 0.2 to $0.8 \mathrm{~V}$ with a scan rate of $0.005 \mathrm{~V} \cdot \mathrm{s}^{-1}$. The corresponding current response plotted over the time is given in the linear sweep voltammogram (LSV) in Figure 4. As it can be seen from the LSV, an oxidation of the silver is observed starting from a potential of $0.45 \mathrm{~V}$. The peak potential is reached at a value of $0.54 \mathrm{~V}$ and oxidation is completed at a potential of about $0.72 \mathrm{~V}$. Here, two peaks can be seen. The first peak at $0.54 \mathrm{~V}$ can be assigned to the oxidation of silver nanoparticles. The second peak can be assigned to the ITO substrate, as it is also seen in the first cycle of blank experiments in the absence of nanoparticles, represented in Supplementary Figure S1.

The comparison of electrochemically obtained current signals with optical CCD dark-field imaging (CCD-DFM) was performed before and after the linear sweep voltammetry measurement. The respective optical snapshots are given in Figures 5A,B. The images were taken at an exposure time of $2,000 \mathrm{~ms}$ and an objective magnification of 100 . From the images it can be seen that particles are homogeneously distributed and immobilized on the ITO electrode (Figure 5A). After oxidation, most of the nanoparticles disappeared, which is indicated by the complete loss in intensity and color information. From the almost dark image, it can be concluded that the particles fully

\section{CCD imaging}

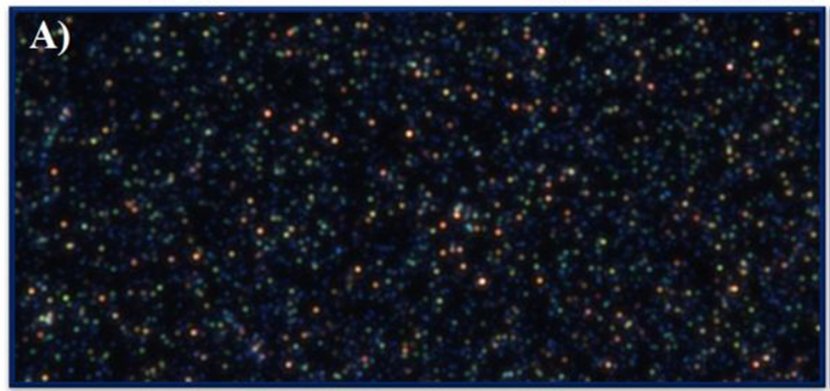

Hyperspectral imaging

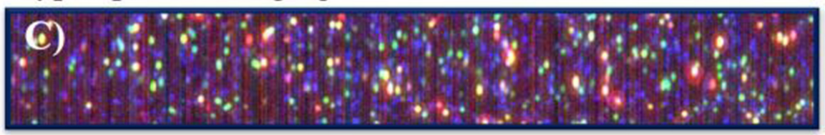

Before the oxidation

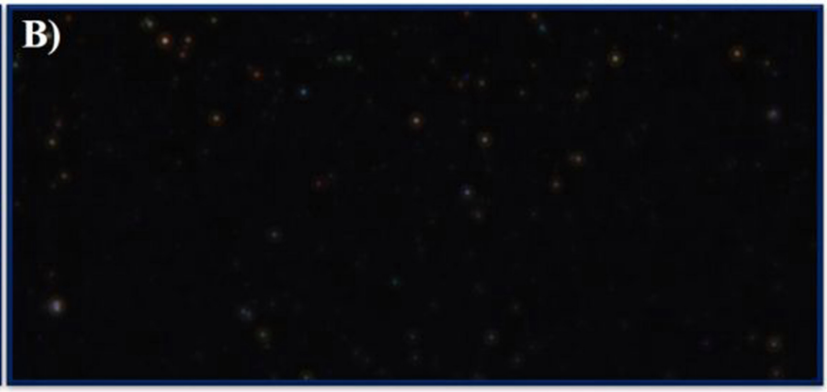

After the oxidation

\section{D)}

FIGURE 5 | (A) DFM-CCD image of AgNPs before the reaction at an exposure time of 2,000 ms. (B) DFM-CCD image of AgNPs after the reaction at an exposure time of 2,000 ms. (C) HSI-DFM image of AgNPs before the reaction at an exposure time of 1,000 ms. (D) HSI-DFM image of AgNPs after the reaction at an exposure time of $1,000 \mathrm{~ms}$. The scale bar is identical for all images. 


\section{A Representative silver nanoparticles}
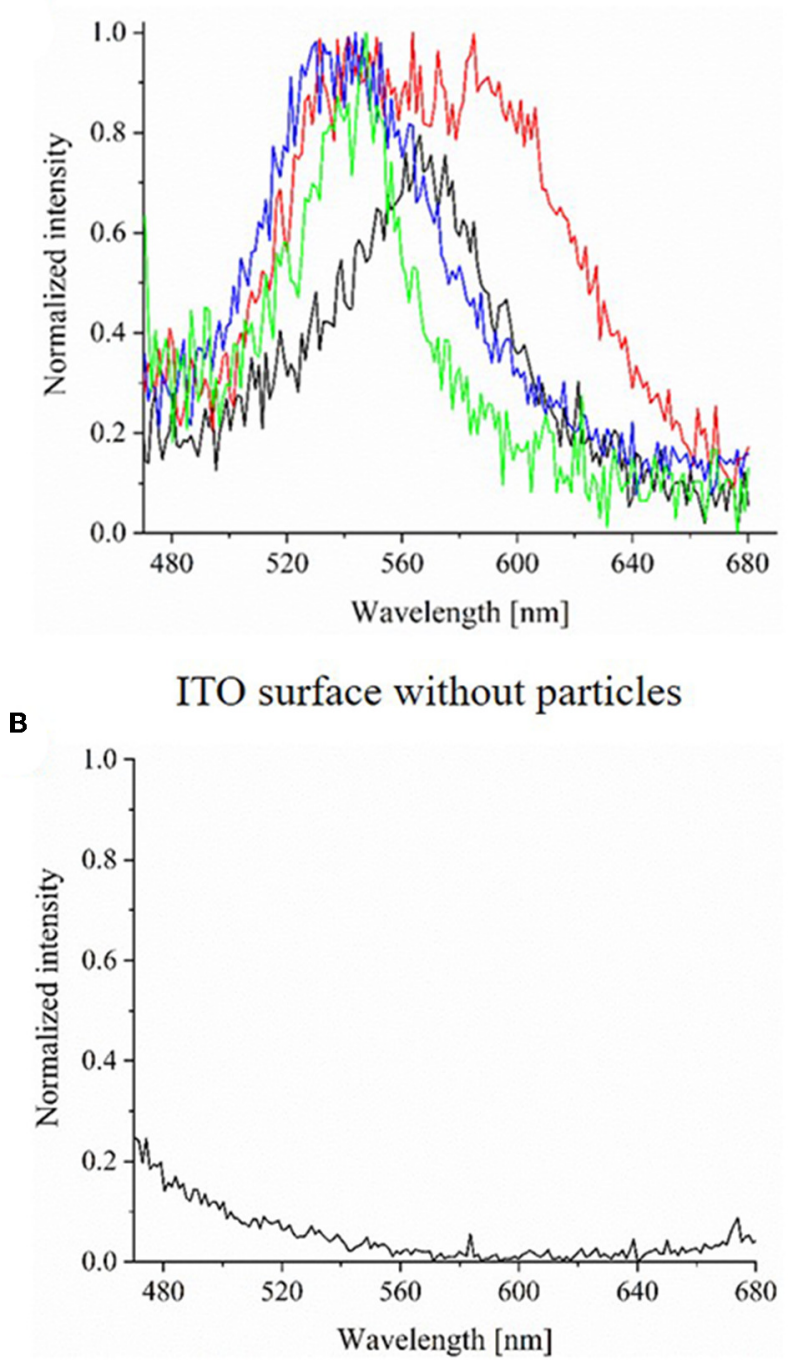

FIGURE 6 | (A) Normalized extinction spectra (exposure time of 1,000 ms) of four representative AgNPs before their electrochemical oxidation on ITO. (B) Normalized extinction spectrum (exposure time of 1,000 ms) of ITO WE without AgNPs.

dissolved during their electrochemical oxidation, while solvated silver cations were formed. Remaining particles are likely not in electrical contact with the ITO electrode (Batchelor-McAuley et al., 2014).

For additional insights, HSI-DFM was performed before and after the reaction. In hyperspectral imaging, single scattering spectra are recorded for each pixel. Based on the spectral information, a color is displayed from a single spectrum. The resulting images are shown in Figures 5C,D and were taken with an exposure time of $1,000 \mathrm{~ms}$. It is indicated that the particles completely vanished and could not be detected. Thus, it is confirmed that the particles dissolved during the oxidation process and did not form a less efficiently scattering insoluble silver species, such as silver oxides (Sundaresan et al., 2018). The optical and hyperspectral images in the absence of silver particles are given in Supplementary Figure S2.

Spectra of single silver nanoparticles can be determined by an extraction of the extinction spectra from the HSI. Spectra of four representative AgNPs are plotted in Figure 6A. All intensities were normalized. As it can be observed, all extinction spectra show a peak maximum at wavelengths between 520 and $600 \mathrm{~nm}$. The blank spectrum of the ITO WE without particles is shown in Figure 6B and lacks this specific peak. Furthermore, also the ITO WE in the absence of silver nanoparticles (Supplementary Figure S3) shows no characteristic peaks or shifts during the electrochemical experiment. From these spectra, it is evident that the characteristic LSPR based extinction peak of the particles vanished during the reaction. Nearly all particles were dissolved during the oxidation, which was shown both by the loss of silver particles in CDD- and HSI and by the corresponding spectral changes.

The reaction process of a single silver nanoparticle during the linear sweep voltammetry can be tracked by HSI. To this end, an individual AgNP was tracked at an exposure time of $5 \mathrm{~s}$ and an objective magnification of 100 . The normalized intensities were plotted against the time in a contour plot, which is given in Figure 7A. The corresponding 3-D illustration of the spectral trace can be found in Supplementary Figure S4. In the intensity trace, it is shown that no spectral change is observed during the first $40 \mathrm{~s}$ of the reaction. In the range of $40-45 \mathrm{~s}$ small changes in the spectra are obtained.

In addition to the spectral shift, the averaged intensity of wavelengths between 580 and $600 \mathrm{~nm}$ was plotted in correlation to the current response over time. The graph is represented in red in Figure 7B, the current is displayed in black.

It is evident, that there is an intensity decrease staring at $40 \mathrm{~s}$, which is in agreement with the spectral shift and the current response. Regarding the spectral intensity information and the current response, the oxidation is finished at a time of 80-85 s. Notably, current response and intensity trace show an exact inverse shape. This demonstrates that electrochemical methods and spectral observation can be directly compared and lead to conclusive results. From the hyperspectral operando imaging it can be concluded that the oxidation and the corresponding dissolution mechanism do not happen instantaneously, but slowly over a longer period of time. For further clarification of the process, representative spectra during the oxidation at experimental times of 45-100 s were plotted against each other with relative (shifted) normalized intensities. The resulting graph is presented in Figure 8.

Two vertical lines at wavelengths of 608 and $673 \mathrm{~nm}$ were added as a guide to the eye of spectral changes. As it can be seen, there is a peak maximum of the extinction spectrum at $608 \mathrm{~nm}$ at $45 \mathrm{~s}$ (black curve), which is based on LSPR light scattering of the initial silver nanoparticle. This peak maximum shifts to $625 \mathrm{~nm}$ at $65 \mathrm{~s}$ (green curve), while the transition happens over peak maxima at $610 \mathrm{~nm}$ (violet curve) and $612 \mathrm{~nm}$ (blue curve) at 50 and $55 \mathrm{~s}$, respectively. This indicates that the particle LSPR changed during the oxidation. It can be assumed that this shift happens due to the change of the particle size/shape. The 
A

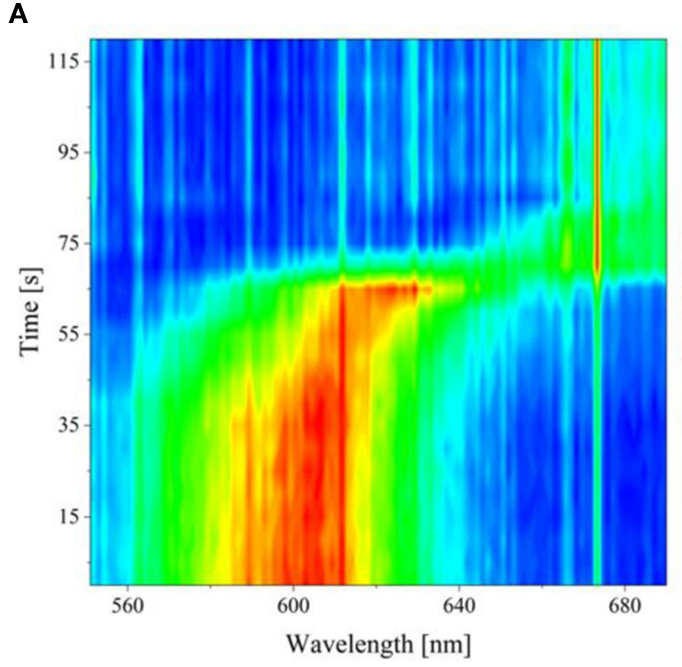

B

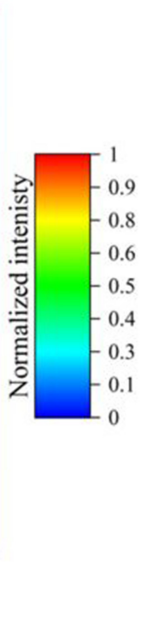

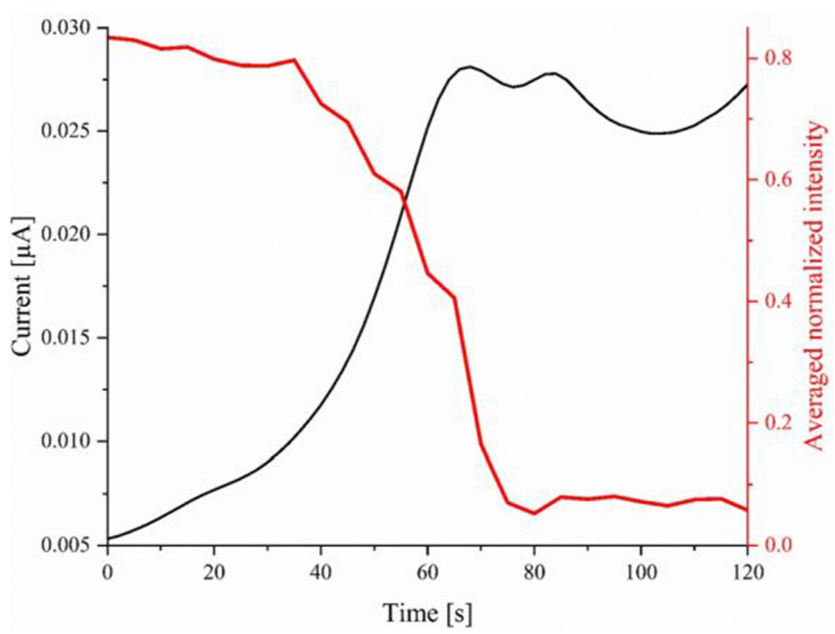

FIGURE 7 | (A) Normalized extinction spectra (exposure time of $5 \mathrm{~s}$ ) of an individual AgNP during its electrochemical oxidation on ITO plotted against the time. (B) Averaged and normalized hyperspectral intensity trace (red) and current (black) correlation plotted against the time.

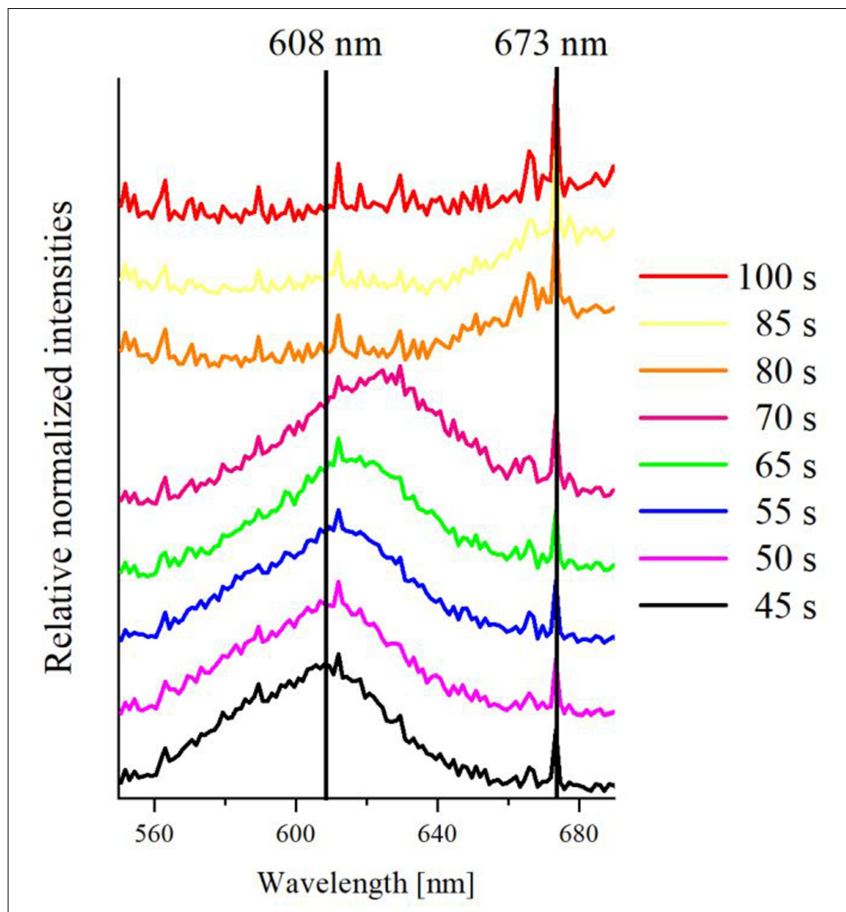

FIGURE 8 | Representative relative and averaged extinction spectra at various times between $45 \mathrm{~s}$ and $100 \mathrm{~s}$ of a single silver nanoparticle at an ITO WE during linear sweep voltammetry, vertically shifted for better comparability.

extinction peak of the LSPR after $70 \mathrm{~s}$ (pink curve) was extremely shifted to $673 \mathrm{~nm}$, broadened and lost most of its scattering intensity. This can be understood as an ongoing dissolution and shrinkage of the particle. After $80 \mathrm{~s}$ (orange curve), the peak shifted to a peak maximum of $673 \mathrm{~nm}$, which is nearly completely vanished at $85 \mathrm{~s}$ (yellow curve). After $100 \mathrm{~s}$ (red curve), no characteristic peak was detected anymore and only the spectrum of the blank ITO glass (Figure 6B) is observed. Since all spectral information were lost, a full conversion coupled with a total dissolution of an individual silver nanoparticle can be concluded. The control experiments were performed in the absence of silver nanoparticles. The blank cyclic voltammogram and the corresponding normalized extinction spectra against the time are given in Supplementary Figures S1, S5, respectively. No spectral change of the scattering was observed, which further confirms the signal change to be caused by the silver particle dissolution. Nevertheless, a small peak at roughly $0.7 \mathrm{~V}$ can be seen in the CV. This can be correlated to the oxidation of the ITO during the first cycle and slightly overlay with the oxidation signal of the silver.

\section{Optical Dark-Field Imaging}

For comparison of the real-time HSI with shorter exposure times, the reaction was observed with optical CCD-imaging. The results are shown in Figure 9.

Optical images were taken before and after the reaction and are given in Figures 9A,B, respectively. Images were taken at an exposure time of $500 \mathrm{~ms}$ and a magnification of the objective of 100. An optical CCD-video was recorded during the reaction with an exposure time of $500 \mathrm{~ms}$, which can be found in Supplementary Material Video 1 and is represented with 20 fps. A multistep chronoamperometric experiment was performed, starting with an applied potential of $-0.4 \mathrm{~V}$ for the first $10 \mathrm{~s}$ and a subsequent switch to $1.2 \mathrm{~V}$ for $300 \mathrm{~s}$. The obtained chronoamperogram (current-time plot) is plotted in Figure 9D. The corresponding chronoamperogram, optical images before and after the electrochemical experiment and the video imaging of the electrochemical experiment in the absence of silver 

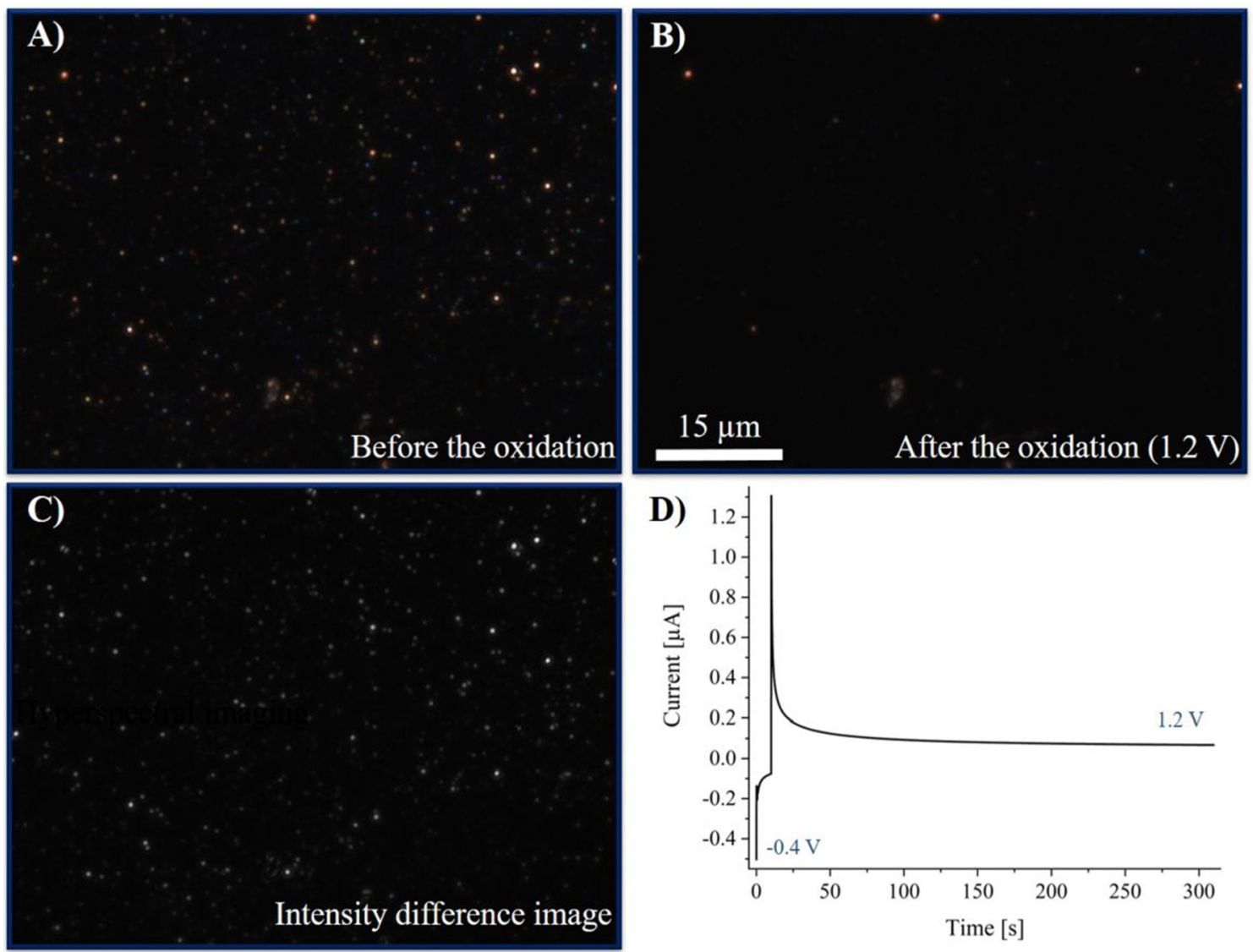

FIGURE 9 | (A) DFM-CCD image of AgNPs before the oxidation at an exposure time of $500 \mathrm{~ms}$. (B) DFM-CCD images of AgNPs after the oxidation at an exposure time of $500 \mathrm{~ms}$. (C) Difference image of (A,B). (D) Chronoamperogram of silver nanoparticles in a $250 \mathrm{mM}$ potassium nitrate solution. The potential was held at $-0.4 \mathrm{~V}$ for $10 \mathrm{~s}$, then the potential was switched to $1.2 \mathrm{~V}$ for $300 \mathrm{~s}$.

nanoparticles are given in Supplementary Figures S6, S7 and Supplementary Material Video S1, respectively.

Previous to the electrochemical experiment, the AgNPs were immobilized as elemental nanoparticles on the electrode. Hence, no change in the color and thus in the LSPR was observed while a potential of $-0.4 \mathrm{~V}$ was applied. From the chronoamperogram, only the negative double layer charging current was observed during the first $10 \mathrm{~s}$. Changing the potential to $1.2 \mathrm{~V}$, a slight decrease in intensity and a simultaneous color change of the particle images was detected. Moreover, a simultaneous steep increase in the current to positive values was obtained. This indicates on the one hand the oxidative current based on the oxidation of AgNPs and on the other hand the inverted double layer charging. As it can be seen in the video, the dissolution does not take place instantaneously, but over several measurement frames. Hence, it can be concluded, that the particle dissolution occurs rather slowly and might happen stepwise.

Optical and hyperspectral results are in accordance with each other. The created difference image (Figure 9C) shows in advance, that nearly all of the particles are dissolved during the reaction. As it can be seen in Figure 9B, a minority of particles (or impurities) was left after the reaction, which might have lacked electrical contact or did not consist of silver. However, these features can be used as an internal reference to ensure that no significant drift of the focus, and, hence, of the detected intensity of imaged particles occurs within the time scale of the experiment. Thus, this change in the extinction of the nanoparticles proves the disappearing of the other AgNPs during the reaction, which otherwise might have arisen from a change in focus of the system.

To validate that the reaction in nitrate electrolytes is indeed slow and not decelerated by the experimental setting (e.g., the resistivity of the ITO WE), a different electrolyte was tested with complexing counter ions. Because silver is preferentially forming $\mathrm{AgCl}$ in the presence of chloride anions (Equation 2), the reaction should be performed in a chloride containing electrolyte of equal ionic strength. In previous works it was shown that silver nanoparticles react nearly instantaneously in $50 \mathrm{mM} \mathrm{KCl}$ on a platinum electrode (Wonner et al., 2018). In this work, a $250 \mathrm{mM}$ solution of $\mathrm{KCl}_{(\mathrm{aq})}$ was used. A multistep chronoamperometric experiment was performed as before with an initial potential of $-0.3 \mathrm{~V}$ for $10 \mathrm{~s}$ and $0.8 \mathrm{~V}$ for $20 \mathrm{~s}$. Optical CCD-imaging was performed during the reaction with an exposure time of $100 \mathrm{~ms}$. Snapshots of the immobilized AgNPs before the electrochemical 

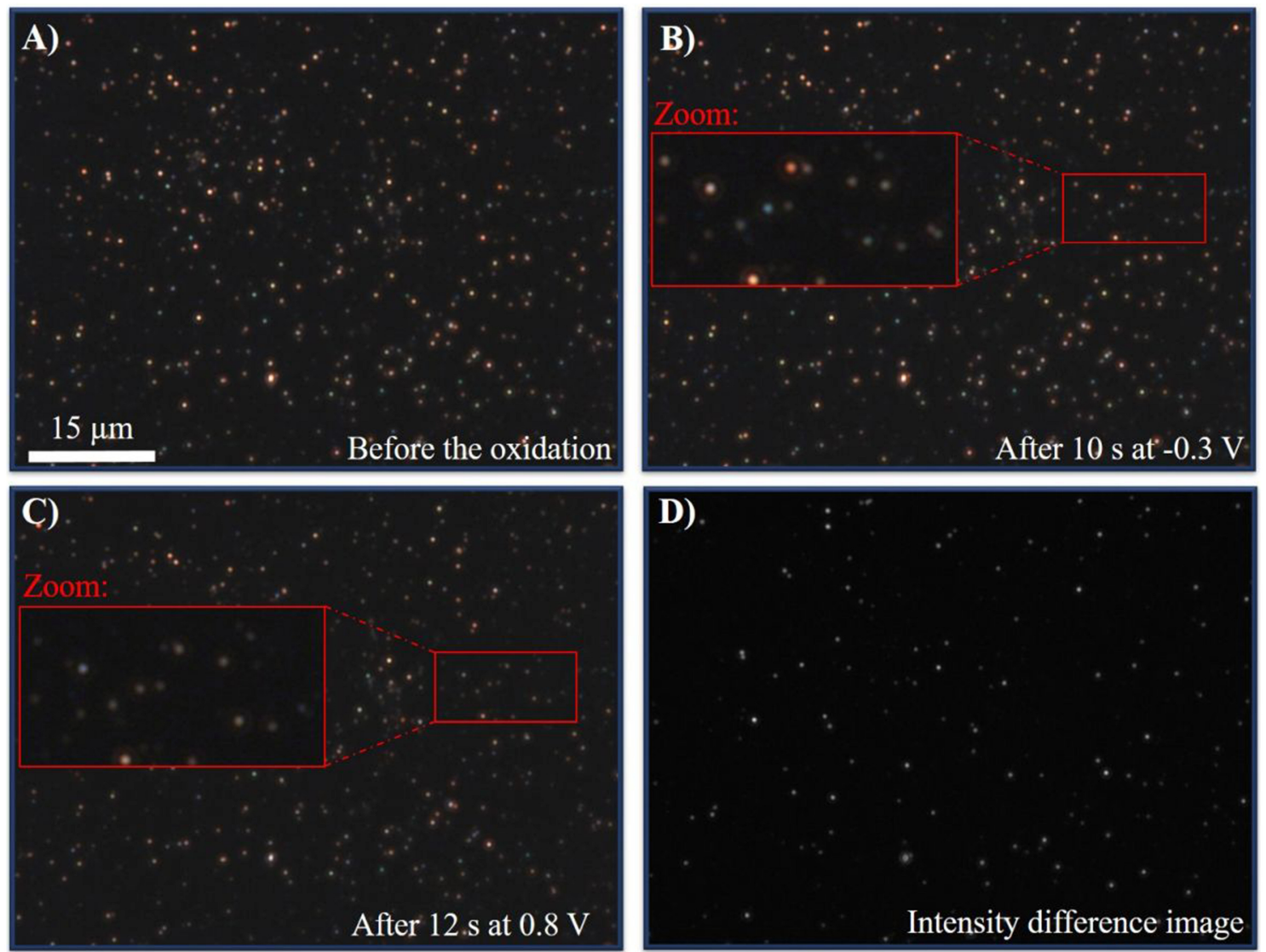

FIGURE 10 | (A) DFM-CCD image of AgNPs before the oxidation at an exposure time of $100 \mathrm{~ms}$. (B) DFM-CCD images of AgNPs after $10.0 \mathrm{~s}$ at -0.3 V at an exposure time of $100 \mathrm{~ms}$. (C) DFM-CCD images of AgNPs after 10.2 s at 0.8 V at an exposure time of $100 \mathrm{~ms}$. (D) Difference image of (B,C).

measurement (A), after $10.0 \mathrm{~s}$ at $-0.3 \mathrm{~V}(\mathrm{~B})$, and after $12 \mathrm{~s}$ at $0.8 \mathrm{~V}(\mathrm{C})$ are given in Figures $10 \mathrm{~A}-\mathrm{C}$, respectively. The optical video imaging is given in the Supplementary Material Video 2. The corresponding chronoamperogram in the presence and absence of silver nanoparticles is displayed in Supplementary Figures S8, S9, the video imaging in the absence of silver particles in chloride containing solutions is given in Supplementary Material Video S2, respectively.

From the video $(0-10 \mathrm{~s})$ and the images Figures 10A,B it is evident that no color or intensity change can be observed during the first $10 \mathrm{~s}$, when a potential of $-0.3 \mathrm{~V}$ was applied. This indicates that elemental silver nanoparticles were immobilized at the surface, which cannot be reduced further. Switching to an oxidative potential of $0.8 \mathrm{~V} \mathrm{(10-30} \mathrm{s} \mathrm{in} \mathrm{the} \mathrm{video} \mathrm{and} \mathrm{Figure} \mathrm{10C,}$ a clear, distinct and instantaneous change in the scattering intensity and color change was observed. This clear and fast shift indicates the oxidation to silver chloride. Comparing the scattering of the particles after $12 \mathrm{~s}$ (Figure 10C) and $10 \mathrm{~s}$ (Figure 10B), a difference image of both states can be created. The resulting difference image is given in Figure 10D. As it can be seen, most of the particles changed their appearance due to the oxidation, which is represented as bright dots in the image. Hence, it can be concluded that within $2 \mathrm{~s}$ most of the particles reacted. This is in agreement with previous studies in chloride solution (Wonner et al., 2018) and much faster than the timescale of the reaction in nitrate containing electrolyte, discussed above.

With this control experiment, it was shown that the oxidation of silver in chloride is not hindered by, for example, insufficient potential drop at the ITO WE. Compared to the dissolution in nitrate, the oxidation in chloride is accelerated and finished after a short time. Hence, it can be concluded that the reaction and coupled dissolution of silver nanoparticles is indeed slowed down in the presence of nitrate anions. Nitrate as a non-complexing counter ion requires the oxidation to solvated silver cations in the solution, which is identified as a significantly slower process in this work that the electrochemical oxidation of silver to silver chloride.

\section{DISCUSSION}

In this work, it was shown that individual immobilized silver nanoparticles can dissolve during electrochemical oxidation in a nitrate containing electrolyte. It was observed that the particles vanished completely after a reaction times of several seconds. Furthermore, the dissolution process seems to be a slow process, 
compared to the oxidation in the presence of chloride anions. The deceleration of the oxidation process seems to be based on the thermodynamic unfavorable silver cation formation in the presence of non-complexing counter ions, in contrast to the silver chloride formation in the presence of precipitating anions.

This study gives insight into the reaction dynamics of individual nanoparticles during their electrochemical oxidation. It can be considered that the oxidation process in nitrate is not an instantaneous and full dissolution, but rather a slow dissolution mechanism. The results give hints that the oxidation during single nanoparticle impacts at a polarized electrode happen over several successive events. Nevertheless, neither a hopping nor a movement of the particles, which was already assumed in literature (Oja et al., 2017; Ustarroz et al., 2017) was observed.

Based on this study it is evident that operando opto- and spectro-electrochemical dark-field microscopy studies are a strong tool to characterize processes at individual nano entities and differentiate the reaction dynamics in various systems. Accordingly, it is anticipated that this approach may be exploited systematically to study electrocatalytic processes at different nanomaterials in the future.

\section{DATA AVAILABILITY STATEMENT}

The raw data supporting the conclusions of this manuscript will be made available by the authors, without undue reservation, to any qualified researcher.

\section{AUTHOR CONTRIBUTIONS}

All authors listed have made a substantial, direct and intellectual contribution to the work, and approved it for publication.

\section{FUNDING}

KW is grateful for a Ph.D. scholarship donated by the German Chemical Industry Fund (FCI Kekule Stipendium). This work was supported by the Research Training group 'Confinement-controlled Chemistry', funded by the
Deutsche Forschungsgemeinschaft (DFG) under Grant GRK2376/331085229. This work was also funded by the Deutsche Forschungsgemeinschaft (DFG, German Research Foundation) under Germany's Excellence Strategy-EXC2033-Projektnummer 390677874. It was also funded by the Ministry of Innovation, Science and Research of North Rhine-Westphalia (NRW Rückkehrerprogramm).

\section{ACKNOWLEDGMENTS}

We thank DFG and BMBF for support of ZEMOS facilities. We also thank Mathies V. Evers for his vivid discussions.

\section{SUPPLEMENTARY MATERIAL}

The Supplementary Material for this article can be found online at: https://www.frontiersin.org/articles/10.3389/fchem. 2019.00912/full\#supplementary-material

3-D HSI plot, Chronoamperogram in the presence of silver nanoparticles in potassium chloride solution, Optical and hyperspectral imaging in the absence of silver nanoparticles, LSV and chronoamperogram's in the absence of silver nanoparticles (PDF).

Supplementary Video 1 | The video shows the dissolution process of silver nanoparticles during its electrochemical oxidation in $250 \mathrm{mM} \mathrm{KNO}_{3}$ at an ITO electrode at a potential of $-0.4 \mathrm{~V}$ for $10 \mathrm{~s}$ and $1.2 \mathrm{~V}$ for $300 \mathrm{~s}$. Images were taken at an exposure time of $500 \mathrm{~ms}$ and an objective magnification of 100 .

Supplementary Video 2 | The video shows the reaction of silver nanoparticles during its electrochemical oxidation in $250 \mathrm{mM} \mathrm{KCl}$ at an ITO electrode at a potential of $-0.3 \mathrm{~V}$ for $10 \mathrm{~s}$ and $0.8 \mathrm{~V}$ for $20 \mathrm{~s}$. Images were taken at an exposure time of $100 \mathrm{~ms}$ and an objective magnification of 100 .

Supplementary Video S1 | The video shows the blank experiment in the absence of silver nanoparticles in $250 \mathrm{mM} \mathrm{KNO}_{3}$ of an ITO electrode at a potential of $-0.4 \mathrm{~V}$ for $10 \mathrm{~s}$ and $1.2 \mathrm{~V}$ for $300 \mathrm{~s}$. Images were taken at an exposure time of $500 \mathrm{~ms}$ and an objective magnification of 100 .

Supplementary Video S2 | The video shows the blank experiment in the absence of silver nanoparticles in $250 \mathrm{mM} \mathrm{KCl}$ of an ITO electrode at a potential of $-0.3 \mathrm{~V}$ for $10 \mathrm{~s}$ and $0.8 \mathrm{~V}$ for $300 \mathrm{~s}$. Images were taken at an exposure time of $100 \mathrm{~ms}$ and an objective magnification of 100.

\section{REFERENCES}

Amendola, V., and Meneghetti, M. (2009). Size evaluation of gold nanoparticles by UV-vis spectroscopy. J. Phys. Chem. C 113, 4277-4285. doi: 10.1021/jp 8082425

Batchelor-McAuley, C., Ellison, J., Tschulik, K., Hurst, P. L., Boldt, R., and Compton, R. G. (2015). In situ nanoparticle sizing with zeptomole sensitivity. Analyst 140, 5048-5054. doi: 10.1039/C5AN00474H

Batchelor-McAuley, C., Martinez-Marrades, A., Tschulik, K., Patel, A. N., Combellas, C., Kanoufi, F., et al. (2014). Simultaneous electrochemical and 3D optical imaging of silver nanoparticle oxidation. Chem. Phys. Lett. 597, 20-25. doi: 10.1016/j.cplett.2014.02.007

Binnig, G., and Rohrer, H. (1983). Scanning tunneling microscopy. Surface Sci. 126, 236-244. doi: 10.1016/0039-6028(83)90716-1

Brasiliense, V., Clausmeyer, J., Berto, P., Tessier, G., Combellas, C., Schuhmann, W., et al. (2018). Monitoring cobalt-oxide single particle electrochemistry with subdiffraction accuracy. Anal. Chem. 90, 7341-7348. doi: 10.1021/acs.analchem.8b00649

Brasiliense, V., Patel, A. N., Martinez-Marrades, A., Shi, J., Chen, Y., Combellas, C., et al. (2016). Correlated electrochemical and optical detection reveals the chemical reactivity of individual silver nanoparticles. J. Am. Chem. Soc. 138, 3478-3483. doi: 10.1021/jacs.5b 13217

Cao, P., Ni, Y., Zou, R., Zhang, L., and Yue, D. (2015). Enhanced catalytic properties of rhodium nanoparticles deposited on chemically modified $\mathrm{SiO}_{2}$ for hydrogenation of nitrile butadiene rubber. RSC Adv. 5, 3417-3424. doi: 10.1039/C4RA11711E

Cheng, W., and Compton, R. G. (2014). Electrochemical detection of nanoparticles by 'nano-impact' methods. Trends Anal. Chem. 58, 79-89. doi: 10.1016/j.trac.2014.01.008

Cheng, W., Stuart, E. J. E., Tschulik, K., Cullen, J. T., and Compton, R. G. (2013). A disposable sticky electrode for the detection of commercial silver NPs in seawater. Nanotechnology 24:505501. doi: 10.1088/0957-4484/24/50/ 505501

Chernousova, S., and Epple, M. (2013). Silver as antibacterial agent. Angew. Chem Int. Ed. 52, 1636-1653. doi: 10.1002/anie.201205923 
El Arrassi, A., Liu, Z., Evers, M. V., Blanc, N., Bendt, G., Saddeler, S., et al. (2019). Intrinsic activity of oxygen evolution catalysts probed at single $\mathrm{CoFe} 2 \mathrm{O} 4$ nanoparticles. J. Am. Chem. Soc. 141, 9197-9201. doi: 10.1021/jacs.9b04516

Evers, M. V., Bernal, M., Roldan Cuenya, B., and Tschulik, K. (2019). Piece by piece-electrochemical synthesis of individual nanoparticles and their performance in ORR electrocatalysis. Angew. Chem. Int. Ed. 58, 8221-8225. doi: 10.1002/anie.201813993

Fang, Y., Wang, W., Wo, X., Luo, Y., Yin, S., Wang, Y., et al. (2014). Plasmonic imaging of electrochemical oxidation of single nanoparticles. J. Am. Chem. Soc. 136, 12584-12587. doi: 10.1021/ja507097y

Ferreira, M., Leite, F. L., de Oliveira, O., and Roz, A. L. (eds.). (2015). Técnicas de Nanocaracterização. Rio de Janeiro: Elsevier.

Grasmik, V., Rurainsky, C., Loza, K., Evers, M. V., Prymak, O., Heggen, M., et al. (2018). Deciphering the surface composition and the internal structure of alloyed silver-gold nanoparticles. Chemistry 24, 9051-9060. doi: 10.1002/chem.201800579

Haiss, W., Thanh, N. T. K., Aveyard, J., and Fernig, D. G. (2007). Determination of size and concentration of gold nanoparticles from UV-vis spectra. Anal. Chem. 79, 4215-4221. doi: 10.1021/ac0702084

Hao, R., Fan, Y., and Zhang, B. (2017). Imaging dynamic collision and oxidation of single silver nanoparticles at the electrode/solution interface. J. Am. Chem. Soc. 139, 12274-12282. doi: 10.1021/jacs.7b06431

Kanokkanchana, K., Saw, E. N., and Tschulik, K. (2018). Nano impact electrochemistry: effects of electronic filtering on peak height, duration and area. ChemElectroChem 5, 3000-3005. doi: 10.1002/celc.201 800738

Kelly, K. L., Coronado, E., Zhao, L. L., and Schatz, G. C. (2003). The optical properties of metal nanoparticles. J. Phys. Chem. B 107, 668-677. doi: 10.1021/jp026731y

Khan, I., Saeed, K., and Khan, I. (2017). Nanoparticles: properties, applications and toxicities. Arab. J. Chem. 12, 908-931. doi: 10.1016/j.arabjc.2017. 05.011

Konopatsky, A. S., Firestein, K. L., Leybo, D. V., Popov, Z. I., Larionov, K. V., Steinman, A. E., et al. (2018). BN nanoparticle/Ag hybrids with enhanced catalytic activity. Catal. Sci. Technol. 8, 1652-1662. doi: 10.1039/C7CY02207G

Kreibig, U. (1974). Electronic properties of small silver particles. J. Phys. F Metal Phys. 4, 999-1014. doi: 10.1088/0305-4608/ 4/7/007

Little, C. A., Batchelor-McAuley, C., Ngamchuea, K., Lin, C., Young, N. P., and Compton, R. G. (2018). Coupled optical and electrochemical probing of silver nanoparticle destruction in a reaction layer. ChemistryOpen 7, 370-380. doi: 10.1002/open.201800048

Mock, J. J., Barbic, M., Smith, D. R., Schultz, D. A., and Schultz, S. (2002). Shape effects in plasmon resonance of individual colloidal silver nanoparticles. J. Chem. Phys. 116, 6755-6759. doi: 10.1063/1.1 462610

Ngamchuea, K., Batchelor-McAuley, C., and Compton, R. G. (2018). Understanding electroanalytical measurements in authentic human saliva leading to the detection of salivary uric acid. Sens Actuat. B Chem. 262, 404-410. doi: 10.1016/j.snb.2018.02.014

Oja, S. M., Robinson, D. A., Vitti, N. J., Edwards, M. A., Liu, Y., White, H. S., et al. (2017). Observation of multipeak collision behavior during the electro-oxidation of single $\mathrm{Ag}$ nanoparticles. J. Am. Chem. Soc. 139, 708-718. doi: 10.1021/jacs. $6 \mathrm{~b} 11143$

Patel, A. N., Martinez-Marrades, A., Brasiliense, V., Koshelev, D., Besbes, M., Kuszelewicz, R., et al. (2015). Deciphering the elementary steps of transportreaction processes at individual $\mathrm{Ag}$ nanoparticles by $3 \mathrm{D}$ superlocalization microscopy. Nano Lett. 15, 6454-6463. doi: 10.1021/acs.nanolett.5 b02921

Qiu, K., Fato, T. P., Wang, P. Y., and Long, Y. T. (2019). Real-time monitoring of the electrochemical reactions on single nanoparticles by dark-field and Raman microscopy. Dalton Trans. 48, 3809-3814. doi: 10.1039/C8DT05141K

Ramos, A. P. (2017). 4 - "Dynamic light scattering applied to nanoparticle characterization," in Nanocharacterization Techniques, eds A. L. Da Róz, M. Ferreira, F. de Lima Leite, and O. N. Oliveira, Jr. (Ribeirão Preto: University of São Paulo; Elsevier), 99-110. doi: 10.1016/B978-0-323-49778-7.00004-7
Robinson, D. A., Liu, Y., Edwards, M. A., Vitti, N. J., Oja, S. M., Zhang, B., et al. (2017). Collision dynamics during the electrooxidation of individual silver nanoparticles. J. Am. Chem. Soc. 139, 16923-16931. doi: 10.1021/jacs.7b09842

Roduner, E. (2006). Size matters: why nanomaterials are different. Chem. Soc. Rev. 35, 583-592. doi: 10.1039/b502142c

Salehi-Khojin, A., Jhong, H. R. M., Rosen, B. A., Zhu, W., Ma, S., Kenis, P. J. A., et al. (2013). Nanoparticle silver catalysts that show enhanced activity for carbon dioxide electrolysis. J. Phys. Chem. C 117, 1627-1632. doi: 10.1021/jp310509z

Saw, E. N., Blanc, N., Kanokkanchana, K., and Tschulik, K. (2018). Time-resolved impact electrochemistry - A new method to determine diffusion coefficients of ions in solution. Electrochim. Acta 282, 317-323. doi: 10.1016/j.electacta.2018. 06.013

Saw, E. N., Grasmik, V., Rurainsky, C., Epple, M., and Tschulik, K. (2016). Electrochemistry at single bimetallic nanoparticles - using nano impacts for sizing and compositional analysis of individual AgAu alloy nanoparticles. Faraday Discuss. 193, 327-338. doi: 10.1039/C6FD00112B

Saw, E. N., Kratz, M., and Tschulik, K. (2017). Time-resolved impact electrochemistry for quantitative measurement of single-nanoparticle reaction kinetics. Nano Res. 10, 3680-3689. doi: 10.1007/s12274-017-1578-3

Shan, X., Díez-Pérez, I., Wang, L., Wiktor, P., Gu, Y., Zhang, L., et al. (2012). Imaging the electrocatalytic activity of single nanoparticles. Nat. Nanotechnol. 7, 668-672. doi: 10.1038/nnano.20 12.134

Sherry, L. J., Chang, S. H., Schatz, G. C., van Duyne, R. P., Wiley, B. J., and Xia, Y. (2005). Localized surface plasmon resonance spectroscopy of single silver nanocubes. Nano Lett. 5, 2034-2038. doi: 10.1021/nl05 15753

Sokolov, S. V., Tschulik, K., Batchelor-McAuley, C., Jurkschat, K., and Compton, R. G. (2015). Reversible or not? Distinguishing agglomeration and aggregation at the nanoscale. Anal. Chem. 87, 10033-10039. doi: 10.1021/acs.analchem.5b02639

Stiles, P. L., Dieringer, J. A., Shah, N. C., and van Duyne, R. P. (2008). Surface-enhanced Raman spectroscopy. Annu. Rev. Anal. Chem. 1, 601-626. doi: 10.1146/annurev.anchem.1.031207.112814

Strickler, A. L., Escudero-Escribano, M. A., and Jaramillo, T. F. (2017). Core-shell Au@metal-oxide nanoparticle electrocatalysts for enhanced oxygen evolution. Nano Lett. 17, 6040-6046. doi: 10.1021/acs.nanolett.7b02357

Sun, L., Wang, W., and Chen, H. Y. (2018). Dynamic nanoparticle-substrate contacts regulate multi-peak behavior of single silver nanoparticle collisions. ChemElectroChem. 5, 2995-2999. doi: 10.1002/celc.2018 00640

Sundaresan, V., Monaghan, J. W., and Willets, K. A. (2018). Visualizing the effect of partial oxide formation on single silver nanoparticle electrodissolution. J. Phys. Chem. C 122, 3138-3145. doi: 10.1021/acs.jpcc.7b11824

Tanner, E. E. L., Sokolov, S. V., Young, N. P., Batchelor-McAuley, C., and Compton, R. G. (2017). Fluorescence electrochemical microscopy. Angew. Chem. Int. Ed. 56, 12751-12754. doi: 10.1002/anie.201707809

Toh, H. S., Batchelor-McAuley, C., Tschulik, K., and Compton, R. G. (2013). Electrochemical detection of chloride levels in sweat using silver nanoparticles. Analyst 138, 4292-4297. doi: 10.1039/c3an00843f

Tschulik, K., Batchelor-McAuley, C., Toh, H. S., Stuart, E. J., and Compton, R. G. (2014). Electrochemical studies of silver nanoparticles. Phys. Chem. Chem. Phys. 16, 616-623. doi: 10.1039/C3CP54221A

Tschulik, K., Palgrave, R. G., Batchelor-McAuley, C., and Compton, R. G. (2013). 'Sticky electrodes' for the detection of silver nanoparticles. Nanotechnology 24:295502. doi: 10.1088/0957-4484/24/29/295502

Ustarroz, J., Kang, M., Bullions, E., and Unwin, P. R. (2017). Impact and oxidation of single silver nanoparticles at electrode surfaces. Chem. Sci. 8, 1841-1853. doi: 10.1039/C6SC04483B

Viñes, F., Gomes, J. R. B., and Illas, F. (2014). Understanding the reactivity of metallic nanoparticles: beyond the extended surface model for catalysis. Chem. Soc. Rev. 43, 4922-4939. doi: 10.1039/C3CS60421G

Wang, H., Lee, H. W., Deng, Y., Lu, Z., Hsu, P. C., Liu, Y., et al. (2015). Bifunctional non-noble metal oxide nanoparticle electrocatalysts through lithium-induced conversion for overall water splitting. Nat. Commun. 6:7261. doi: $10.1038 /$ ncomms 8261 
Wang, Z. L. (2000). Transmission electron microscopy of shape-controlled nanocrystals and their assemblies. J. Phys. Chem. B 104, 1153-1175. doi: $10.1021 /$ jp993593c

Weber, M. L., Wilson, A. J., and Willets, K. A. (2015). Characterizing the spatial dependence of redox chemistry on plasmonic nanoparticle electrodes using correlated super-resolution surface-enhanced Raman scattering imaging and electron microscopy. J. Phys. Chem. C 119, 18591-18601. doi: 10.1021/acs.jpcc.5b05715

Welch, C. M., and Compton, R. G. (2006). The use of nanoparticles in electroanalysis. Anal. Bioanal. Chem. 384, 601-619. doi: 10.1007/s00216-005-0230-3

Willets, K. A., and van Duyne, R. P. (2007). Localized surface plasmon resonance spectroscopy and sensing, Annu. Rev. Phys. Chem. 58, 267-297. doi: 10.1146/annurev.physchem.58.032806.104607

Willets, K. A., Wilson, A. J., Sundaresan, V., and Joshi, P. B. (2017). Super-resolution imaging and plasmonics. Chem. Rev. 117, 7538-7582. doi: 10.1021/acs.chemrev.6b00547

Wilson, A. J., Marchuk, K., and Willets, K. A. (2015). Imaging Electrogenerated chemiluminescence at single gold nanowire electrodes. Nano Lett. 15, 6110-6115. doi: 10.1021/acs.nanolett.5b02383

Wilson, A. J., and Willets, K. A. (2016). Molecular plasmonics. Annu. Rev. Anal. Chem. 9, 27-43. doi: 10.1146/annurev-anchem-071015-041612

Wong, K. K. Y., Cheung, S. O., Huang, L., Niu, J., Tao, C., Ho, C. M., et al. (2009). Further evidence of the anti-inflammatory effects of silver nanoparticles. ChemMedChem 4, 1129-1135. doi: 10.1002/cmdc.200900049
Wonner, K., Evers, M. V., and Tschulik, K. (2018). Simultaneous optoand spectro-electrochemistry. J. Am. Chem. Soc. 140, 12658-12661. doi: $10.1021 /$ jacs.8b02367

Wonner, K., Evers, M. V., and Tschulik, K. (2019). The electrochemical dissolution of single silver nanoparticles enlightened by hyperspectral dark-field microscopy. Electrochim. Acta 301, 458-464. doi: 10.1016/j.electacta.2019.01.129

Yang, S., Bachman, R. E., Feng, X., and Müllen, K. (2013). Use of organic precursors and graphenes in the controlled synthesis of carbon-containing nanomaterials for energy storage and conversion. Acc. Chem. Res. 46, 116-128. doi: 10.1021/ar3001475

Zhu, J., Holmen, A., and Chen, D. (2013). Carbon nanomaterials in catalysis. ChemCatChem 5, 378-401. doi: 10.1002/cctc.201200471

Conflict of Interest: The authors declare that the research was conducted in the absence of any commercial or financial relationships that could be construed as a potential conflict of interest.

Copyright (c) 2020 Wonner, Rurainsky and Tschulik. This is an open-access article distributed under the terms of the Creative Commons Attribution License (CC BY).

The use, distribution or reproduction in other forums is permitted, provided the original author(s) and the copyright owner(s) are credited and that the original publication in this journal is cited, in accordance with accepted academic practice. No use, distribution or reproduction is permitted which does not comply with these terms. 